\title{
ON A UNIQUELY SOLVABLE INTEGRAL EQUATION IN A MIXED DIRICHLET-NEUMANN PROBLEM OF ACOUSTIC SCATTERING
}

\author{
BY \\ P. A. KRUTITSKII \\ Dept. of Mathematics, Faculty of Physics, Moscow State University, Moscow 119899, Russia
}

\begin{abstract}
The mixed Dirichlet-Neumann problem for the Helmholtz equation in the exterior of several bodies (obstacles) is studied in 2 and 3 dimensions. The problem is investigated by a special modification of the boundary integral equation method. This modification can be called the "method of interior boundaries", because additional boundaries are introduced inside scattering bodies, where the Neumann boundary condition is given. The solution of the problem is obtained in the form of potentials on the whole boundary. The density in the potentials satisfies the uniquely solvable Fredholm equation of the second kind and can be computed by standard codes. In fact, our method holds for any positive wave numbers. The Neumann and Dirichlet problems are particular cases of our problem.
\end{abstract}

1. Introduction. We study the mixed Dirichlet-Neumann problem for the propagative Helmholtz equation in the exterior of several bodies (obstacles) in 2 and 3 dimensions. The Dirichlet boundary condition is given on some bodies and the Neumann boundary condition is specified on the rest. Similar problems model, for example, scattering acoustic waves by several obstacles. The Dirichlet and Neumann problems are particular cases of our problem. The aim of the present paper is to suggest a new approach to reduction of the mixed problem for the propagative Helmholtz equation to the uniquely solvable Fredholm integral equation of the second kind. This equation is very useful in applications, because its numerical solution can be obtained by standard codes. To derive this equation we put additional boundaries inside obstacles with the Neumann boundary condition and specify appropriate boundary conditions on the additional boundaries. The modified problem with additional boundaries has no more than one solution. We look for a solution of the problem in the form of a single layer potential on additional boundaries and on the obstacles with the Neumann boundary condition. According to [17, 12, 2], a linear combination of single and double layer potentials is taken on the obstacles with

Received August 16, 1999.

2000 Mathematics Subject Classification. Primary 45B05, 35J25, 35J05, 31B20.

E-mail address: krutitsk@math.phys.msu.su

(C)2001 Brown University 
Dirichlet boundary condition. Substituting the solution in the form of potentials into the boundary condition, we obtain an integral equation on the whole boundary. Next we verify that the integral equation obtained is a uniquely solvable Fredholm equation of the second kind.

Let us compare our approach with two classical methods. In $[4,5,21,22]$ it was suggested to put an infinite number of point sources inside obstacles with the Neumann boundary condition. This method enables us to prove a formal solvability theorem, but it was not widely used in applications, since it is very hard to take into account an infinite number of point sources when finding a numerical solution. In our approach we exchange point sources for distributed sources in the form of additional boundaries. In $[12,17]$ it was proposed to look for a solution of the problem in the form of a linear combination of single and double layer potentials on the obstacles with the Neumann boundary condition. The hypersingular integral equation has been obtained on the surface of these obstacles. The numerical analysis of hypersingular integral equations requires special approaches [3, $11,13,14,15,18,19,20]$, and it is much more complicated than the numerical treatment of the uniquely solvable Fredholm equation obtained in our method. In addition, the normal derivative of the double layer potential may not exist, while the classical solution exists. Therefore, the solution cannot be represented as a sum of single and double layer potentials on obstacles with the Neumann boundary condition in certain cases.

Our method holds for any wave number $k \in\left[0, k_{0}\right]$ in a 3 -D case and for any $k \in\left(0, k_{0}\right]$ in a 2-D case, where $k_{0}$ is an arbitrary fixed positive number. In fact, our method holds for all $k$, which may be used for computations in practical problems, since $k_{0}$ can be taken large enough, i.e., as large as is necessary. In addition, the case of sufficiently large $k$ is not interesting in diffraction theory, since if $k \rightarrow \infty$, the diffraction is absent and waves are subject to the laws of geometric optics such as reflection and refraction. The problems on scattering waves by a finite number of 2-D non-closed screens (open arcs) were reduced to the uniquely solvable Fredholm integral equations in $[6,7,8,9,10]$.

2. Formulation of the problem. Let $x=\left(x_{1}, \ldots, x_{n}\right) \in \mathbb{R}^{m}$ for $m=2$ or $m=3$, and $\Delta$ is a Laplacian in $\mathbb{R}^{m}$. We consider an exterior open multiply-connected domain $\mathcal{D} \subset \mathbb{R}^{m}$ with the boundary $\Gamma=\Gamma^{1} \cup \Gamma^{2}$, where

$$
\Gamma^{1}=\bigcup_{n=1}^{N_{1}} \Gamma_{n}^{1} \in C^{2, \lambda}, \quad \Gamma^{2}=\bigcup_{n=1}^{N_{2}} \Gamma_{n}^{2} \in C^{2,0}, \quad \lambda \in(0,1]
$$

and $\Gamma_{1}^{1}, \ldots, \Gamma_{N_{1}}^{1}, \Gamma_{1}^{2}, \ldots, \Gamma_{N_{2}}^{2}$ are simple closed surfaces if $m=3$ or curves if $m=2$ without common points. Each surface (curve) $\Gamma_{n}^{j}$ bounds an interior single-connected open domain $\mathcal{D}_{n}^{j}\left(n=1, \ldots, N_{j} ; j=1,2\right)$. Let $\mathbf{n}_{x}$ be a unit normal vector to $\Gamma$ at $x \in \Gamma$. The vector $\mathbf{n}_{x}$ is an outward normal to $\mathcal{D}$. Consider $\Gamma_{n}^{j}$ as a double-sided surface (curve). By $\left(\Gamma_{n}^{j}\right)^{-}$we denote that side of $\Gamma_{n}^{j}$ which we observe when facing towards the normal's tips. The opposite side will be called $\left(\Gamma_{n}^{j}\right)^{+}$. Accordingly, $\Gamma^{ \pm}=\left(\Gamma^{1}\right)^{ \pm} \cup\left(\Gamma^{2}\right)^{ \pm}$, $\left(\Gamma^{j}\right)^{ \pm}=\bigcup_{n=1}^{N_{j}}\left(\Gamma_{n}^{j}\right)^{ \pm}, j=1,2$. 
We say that the function $W(x)$ defined in $\overline{\mathcal{D}}$ belongs to the smoothness class $\mathbf{K}$ if

1) $W(x) \in C^{0}(\overline{\mathcal{D}}) \cap C^{2}(\mathcal{D})$,

2) there exists a uniform for all $x \in\left(\Gamma^{2}\right)^{+}$limit of $\left(\mathbf{n}_{x}, \nabla_{\bar{x}} W(\bar{x})\right)$ as $\bar{x} \in \mathcal{D}$ tends to $x \in\left(\Gamma^{2}\right)^{+}$along the normal $\mathbf{n}_{x}$,

3) $\nabla W(x)$ can be continuously extended on $\left(\Gamma^{1}\right)^{+}$from the domain $\mathcal{D}$.

Let us formulate the exterior mixed Dirichlet-Neumann problem for the propagative Helmholtz equation in the domain $\mathcal{D} \subset \mathbb{R}^{m}$ with $m=3$ or $m=2$.

Problem V. To find a function $W(x)$ of the class $\mathbf{K}$, so that $W(x)$ satisfies the Helmholtz equation in $\mathcal{D}$,

$$
\Delta W(x)+k^{2} W(x)=0, \quad k=\operatorname{Re} k=\text { const } \geq 0,
$$

satisfies the boundary conditions

$$
\begin{aligned}
\left.W(x)\right|_{x \in\left(\Gamma^{1}\right)^{+}} & =\left.f(x)\right|_{x \in \Gamma^{1}}, \\
\left.\frac{\partial W(x)}{\partial \mathbf{n}_{x}}\right|_{x \in\left(\Gamma^{2}\right)^{+}} & =\left.f(x)\right|_{x \in \Gamma^{2}}
\end{aligned}
$$

and meets the radiation conditions at infinity,

$$
W=O\left(|x|^{(1-m) / 2}\right), \quad \frac{\partial W}{\partial|x|}-i k W=o\left(|x|^{(1-m) / 2}\right)
$$

as $|x|=\sqrt{x_{1}^{2}+\cdots+x_{m}^{2}} \rightarrow \infty$. The function $f(x)$ is given.

All conditions of the problem must be satisfied in the classical sense. By $\partial W(x) / \partial \mathbf{n}_{x}$ on $\left(\Gamma^{2}\right)^{+}$we mean the limit ensured in the point 2) of the definition of the smoothness class K. Problem V transforms to the Neumann or Dirichlet problem if $\Gamma^{1}=\varnothing$ or $\Gamma^{2}=\varnothing$ respectively.

The theorem holds.

THEOREM 1. There is no more than one solution of the problem $\mathbf{V}$.

Proof. Let $W_{0}(x)$ be an arbitrary solution of the homogeneous problem V. Our aim is to show that $W_{0}(x) \equiv 0$ in $\mathcal{D}$.

By $C_{r}$ we denote a ball (circle if $m=2$ ) of the large radius $r$ with the center in the origin. By $\bar{W}_{0}(x)$ we denote a function which is complex conjugate to $W_{0}(x)$. Clearly, $\bar{W}_{0}(x)$ belongs to the class $\mathbf{K}$. We envelop $\Gamma_{1}^{1}, \ldots, \Gamma_{N_{1}}^{1}, \Gamma_{1}^{2}, \ldots, \Gamma_{N_{2}}^{2}$ by closed equidistant surfaces (contours) [24] lying in domain $\mathcal{D}$ and write an energy identity for the domain bounded by these surfaces (contours) and $C_{r}$. Then we allow surfaces (contours) to tend to $\Gamma^{+}$and $r$ to tend to infinity. Using smoothness of $W_{0}(x)$ ensured by the class $\mathbf{K}$ we get

$$
\begin{aligned}
\lim _{r \rightarrow \infty} & \left(\left\|\nabla W_{0}\right\|_{L_{2}\left(C_{r} \cap \mathcal{D}\right)}^{2}-k^{2}\left\|W_{0}\right\|_{L_{2}\left(C_{r} \cap \mathcal{D}\right)}^{2}\right) \\
& =\int_{\Gamma^{+}} \bar{W}_{0}(x) \frac{\partial W_{0}(x)}{\partial \mathbf{n}_{x}} d s+\lim _{r \rightarrow \infty} \int_{\partial C_{r}} \bar{W}_{0}(x) \frac{\partial W_{0}(x)}{\partial r} d s \\
& =i k \lim _{r \rightarrow \infty} \int_{\partial C_{r}}\left|W_{0}(x)\right|^{2} d s,
\end{aligned}
$$

where the conditions (2.3) and (2.2) were used. By $\int \cdots d s$ we denote the surface (curvilinear) integral of the 1-st kind. If $k=0$ in (2.4), then $\nabla W_{0}(x) \equiv 0, W_{0}(x) \equiv$ const in 
$\mathcal{D}$ and from conditions $(2.3)$ at infinity we get $W_{0}(x) \equiv 0$ in $\mathcal{D}$. Further on we assume that $k>0$ and take the imaginary part in (2.4). Then we obtain

$$
\lim _{r \rightarrow \infty} \int_{\partial C_{r}}\left|W_{0}(x)\right|^{2} d s=0 .
$$

It follows from the Rellich lemma $[2,25]$ that $W_{0}(x) \equiv 0$ for $x \in \mathcal{D}$. Hence the homogeneous problem $\mathbf{V}$ has only a trivial solution for any $k \geq 0$, and the theorem is proved due to the linearity of the problem $\mathbf{V}$.

3. The modified problem. In this section we consider the modification of the problem $\mathbf{V}$, which will be called $\mathbf{V}_{0}$. Recall that in our notation, $m=3$ and $m=2$ correspond to 3 -D and 2-D cases respectively, and $\partial \mathcal{D}_{n}^{2}=\Gamma_{n}^{2}\left(n=1, \ldots, N_{2}\right), \partial \mathcal{D}_{n}^{1}=\Gamma_{n}^{1}$ $\left(n=1, \ldots, N_{1}\right)$. In each domain $\mathcal{D}_{n}^{2}$ we consider a simple closed surface if $m=3$ or curve if $m=2$ of class $C^{2,0}$ and denote it by $\gamma_{n}\left(n=1, \ldots, N_{2}\right)$. Suppose that $\gamma_{n}$ bounds the interior open single connected domain $\mathcal{D}_{n}^{*} \subset \mathcal{D}_{n}^{2}$. The surfaces (curves) $\gamma_{n}$ are chosen in such a way that for any $k$ from the set $\mathcal{I} \subset[0, \infty)$ the following Dirichlet problem in $\mathcal{D}_{n}^{*}$ :

$$
\left\{\begin{array}{l}
u(x) \in C^{0}\left(\overline{\mathcal{D}_{n}^{*}}\right) \cap C^{2}\left(\mathcal{D}_{n}^{*}\right), \\
\Delta u(x)+k^{2} u(x)=0, \quad x \in \mathcal{D}_{n}^{*}, \\
\left.u(x)\right|_{x \in \gamma_{n}}=0
\end{array}\right.
$$

has only the trivial solution $\left(n=1, \ldots, N_{2}\right)$.

Clearly, surfaces (curves) $\gamma_{n}$ can be chosen in different ways. For example, let

$$
k \in \mathcal{I}=\left[0, k_{0}\right],
$$

where $k_{0}$ is an arbitrary fixed positive number. For this set $\mathcal{I}$, as $\gamma_{n}$ we can take an arbitrary sphere if $m=3$ or an arbitrary circumference if $m=2$ lying in $\mathcal{D}_{n}^{2}$ with the radius $r$ satisfying the estimation

$$
\begin{aligned}
& r<\frac{\pi}{k_{0}} \quad \text { if } m=3, \\
& r<\frac{2.40475}{k_{0}} \quad \text { if } m=2 .
\end{aligned}
$$

Let us show that the problem (3.1) has only the trivial solution for any $k$ in (3.2). We consider the spectral problem in $\mathcal{D}_{n}^{*}$

$$
\left\{\begin{array}{l}
U(x) \in C^{0}\left(\overline{\mathcal{D}_{n}^{*}}\right) \cap C^{2}\left(\mathcal{D}_{n}^{*}\right), \\
\Delta U(x)+\lambda^{2} U(x)=0, \quad x \in \mathcal{D}_{n}^{*}, \\
\left.U(x)\right|_{x \in \gamma_{n}}=0 .
\end{array}\right.
$$

The eigenvalues of (3.4) are positive and can be numbered in ascending order [1, p. 298]. The first eigenvalue of the ball is $\lambda_{1}=\pi / r$. The first eigenvalue of the circle is $\lambda_{1}=c / r$, where $c \simeq 2.4048$ is the least positive root of the equation $J_{0}(z)=0$. Here $J_{0}(z)$ is the Bessel function of index zero [1, p. 301], [24]. If $\lambda<\lambda_{1}(r)$ then the problem (3.4) has only a trivial solution. Consequently, the inequality $k_{0}<\lambda_{1}(r)$ ensures that the problem (3.1) has only the trivial solution for any $k$ in (3.2). This inequality leads to (3.3). 
Instead of the ball (circle) we can take as $\mathcal{D}_{n}^{*}$ an arbitrary single connected domain in $\mathcal{D}_{n}$, the diameter $d$ of which satisfies the estimation

$$
d<\ln \left(1+\frac{1}{2 k_{0}^{2}}\right) .
$$

Then the problem (3.1) has only the trivial solution for any $k$ in (3.2). This statement results from [2, Sect. 3.4, Lemma 3.26].

Thus, below we suppose that the surfaces (curves) $\gamma_{1}, \ldots, \gamma_{N_{2}}$ are chosen in the following way.

$$
\left\{\begin{array}{l}
\text { Each } \gamma_{n}\left(n=1, \ldots, N_{2}\right) \text { is a simple closed surface if } m=3 \\
\text { or a curve if } m=2, \text { such that } \gamma_{n} \subset \mathcal{D}_{n}^{2}, \gamma_{n} \text { is of class } C^{2,0} \\
\text { and the problem }(3.1) \text { has only a trivial solution for any } k \\
\text { belonging to the set } \mathcal{I}, \text { where } \mathcal{I} \subset[0, \infty) \text { in case } m=3 \text { and } \\
\mathcal{I} \subset(0, \infty) \text { in case } m=2 .
\end{array}\right.
$$

Consider also one more assumption, where $\gamma_{1}, \ldots, \gamma_{N_{2}}$ are taken constructively.

$$
\left\{\begin{array}{l}
\text { Let } k_{0} \text { be an arbitrary fixed positive number, and let } \gamma_{n} \\
\left(n=1, \ldots, N_{2}\right) \text { be subject to one of two conditions: } \\
1) \gamma_{n} \text { is a sphere if } m=3 \text { or a circumference if } m=2, \\
\gamma_{n} \subset \mathcal{D}_{n}^{2}, \text { and the radius of } \gamma_{n} \text { meets inequality }(3.3) ; \\
\text { 2) } \gamma_{n} \text { is a simple closed surface if } m=3 \text { or a curve if } m=2, \\
\gamma_{n} \text { is of class } C^{2,0}, \gamma_{n} \subset \mathcal{D}_{n}^{2}, \text { and the diameter of } \gamma_{n} \text { meets } \\
\text { inequality (3.5). }
\end{array}\right.
$$

As indicated above, if (3.7) holds, then (3.6) is satisfied with $\mathcal{I}=\left[0, k_{0}\right]$ in case $m=3$ and with $\mathcal{I}=\left(0, k_{0}\right]$ in case $m=2$.

We put $\gamma=\bigcup_{n=1}^{N_{2}} \gamma_{n}$ and introduce the unit normal vector $\mathbf{n}_{x}$ to $\gamma$ at $x \in \gamma$. If $x \in \gamma_{n}$, then the vector $\mathbf{n}_{x}$ is an outward normal to the domain $\mathcal{D}_{n}^{*}$ bounded by $\gamma_{n}$. Consider $\gamma_{n}$ as a double-sided surface (curve). By $\gamma_{n}^{-}$we denote that side of $\gamma_{n}$ which we observe when facing towards the normal's tips. The opposite side of $\gamma_{n}$ will be called $\gamma_{n}^{+}$. Set $\gamma^{ \pm}=\bigcup_{n=1}^{N_{2}} \gamma_{n}^{ \pm}$and $\mathcal{D}^{0}=\bigcup_{n=1}^{N_{2}}\left(\mathcal{D}_{n}^{2} \backslash\left(\mathcal{D}_{n}^{*} \cup \gamma_{n}\right)\right), \mathcal{D}^{1}=\bigcup_{n=1}^{N_{1}} \mathcal{D}_{n}^{1}$.

We say that the function $W(x)$ defined in $\mathbb{R}^{m} \backslash \mathcal{D}^{1}$ belongs to the class of smoothness $\mathbf{K}_{0}$ if

1) $W(x) \in C^{0}\left(\overline{\mathbb{R}^{m} \backslash \mathcal{D}^{1}}\right) \cap C^{2}\left(\mathbb{R}^{m} \backslash\left(\mathcal{D}^{1} \cup \Gamma \cup \gamma\right)\right)$,

2) conditions 2 ), 3) of the definition of the class $\mathbf{K}$ hold,

3) there exists the uniform for all $x \in\left(\Gamma^{2}\right)^{-} \cup \gamma^{-}$limit of $\left(\mathbf{n}_{x}, \nabla_{\bar{x}} W(\bar{x})\right)$ as $\bar{x} \in \mathcal{D}^{0}$ tends to $x \in\left(\Gamma^{2}\right)^{-} \cup \gamma^{-}$along the normal $\mathbf{n}_{x}$.

Clearly, any function of class $\mathbf{K}_{0}$ belongs to the class $\mathbf{K}$, that is, $\mathbf{K}_{0} \subset \mathbf{K}$.

Now we formulate the modified problem, which we call $\mathbf{V}_{0}$.

Problem $\mathbf{V}_{0}$. In the assumption that condition (3.6) holds we must find a function $W(x)$ of the class $\mathbf{K}_{0}$, so that $W(x)$ satisfies the Helmholtz equation (2.1) in $\mathbb{R}^{m} \backslash\left(\mathcal{D}^{1} \cup\right.$ $\Gamma \cup \gamma)$, meets the radiation conditions (2.3), satisfies the boundary conditions (2.2) and the additional homogeneous boundary condition on $\gamma^{-}$

$$
\left.\left(\frac{\partial W(x)}{\partial \mathbf{n}_{x}}-i W(x)\right)\right|_{x \in \gamma^{-}}=0 .
$$


By $\partial W(x) / \partial \mathbf{n}_{x}$ on $\gamma^{-}$we mean the limit ensured in condition 3) of the definition of the smoothness class $\mathbf{K}_{0}$. All conditions of the problem $\mathbf{V}_{0}$ must be satisfied in the classical sense.

Clearly, any solution of the problem $\mathbf{V}_{0}$ is a solution of the problem $\mathbf{V}$.

Let us prove the uniqueness theorem.

Theorem 2. If condition (3.6) holds, then for any $k \in \mathcal{I}$ the problem $\mathbf{V}_{0}$ has no more than one solution.

Proof. Let $W_{0}(x)$ be a solution of the homogeneous problem $\mathbf{V}_{0}$. Our aim is to show that $W_{0}(x) \equiv 0$. As noted above, $W_{0}(x)$ satisfies the homogeneous problem $\mathbf{V}$. According to Theorem 1:

$$
W_{0}(x) \equiv 0, \quad x \in \mathcal{D} .
$$

It follows from the definition of the class $\mathbf{K}_{0}$ that $W_{0}(x)$ is continuous across $\Gamma^{2}$. So, $W_{0}(x)$ satisfies the following homogeneous boundary value problem in $\mathcal{D}^{0}$ :

$$
\begin{gathered}
\Delta W_{0}(x)+k^{2} W_{0}(x)=0, \quad x \in \mathcal{D}^{0}=\bigcup_{n=1}^{N_{2}}\left(\mathcal{D}_{n}^{2} \backslash\left(\mathcal{D}_{n}^{*} \cup \gamma_{n}\right)\right), \\
\left.W_{0}(x)\right|_{x \in \Gamma^{2}}=0 \\
\left.\left(\frac{\partial W_{0}(x)}{\partial \mathbf{n}_{x}}-i W_{0}(x)\right)\right|_{x \in \gamma^{-}}=0 .
\end{gathered}
$$

We construct equidistant surfaces (curves) in $\mathcal{D}^{0}$ for boundaries $\Gamma^{2}$ and $\gamma$, write energy equalities in domains bounded by these surfaces (curves) and tend these surfaces (curves) to the boundaries [24]. Using the smoothness properties, ensured by the class $\mathbf{K}_{0}$, we obtain

$$
\begin{aligned}
-\left\|\nabla W_{0}\right\|_{L_{2}\left(\mathcal{D}^{0}\right)}^{2}+k^{2}\left\|W_{0}\right\|_{L_{2}\left(\mathcal{D}^{0}\right)} & =\int_{\left(\Gamma^{2}\right)-\cup \gamma^{-}} \bar{W}_{0}(x) \frac{\partial W_{0}(x)}{\partial \mathbf{n}_{x}} d s \\
& =\int_{\gamma^{-}} \bar{W}_{0}(x) \frac{\partial W_{0}(x)}{\partial \mathbf{n}_{x}} d s \\
& =i \int_{\gamma}\left|W_{0}(x)\right|^{2} d s,
\end{aligned}
$$

where we applied the boundary conditions $(3.10 \mathrm{~b}, \mathrm{c})$. Taking the imaginary part in the latter identity we have

$$
\int_{\gamma}\left|W_{0}(x)\right|^{2} d s=0 .
$$

Therefore

$$
\left.W_{0}(x)\right|_{x \in \gamma}=0
$$

and, thanks to $(3.10 \mathrm{c})$,

$$
\left.\frac{\partial W_{0}(x)}{\partial \mathbf{n}_{x}}\right|_{x \in \gamma^{-}}=0
$$


The function $W_{0}(x)$ is continuous across $\gamma$ since $W_{0}(x) \in \mathbf{K}_{0}$. Taking into account (3.11) we observe that the function $W_{0}(x)$ satisfies the following Dirichlet problem in each domain $\mathcal{D}_{n}^{*}\left(n=1, \ldots, N_{2}\right)$ :

$$
\begin{gathered}
\Delta W_{0}(x)+k^{2} W_{0}(x)=0, \quad x \in \mathcal{D}_{n}^{*}, \\
\left.W_{0}(x)\right|_{x \in \gamma_{n}}=0 .
\end{gathered}
$$

It follows from the condition (3.6) that

$$
W_{0}(x) \equiv 0, \quad x \in \mathcal{D}_{n}^{*} \quad\left(n=1, \ldots, N_{2}\right) .
$$

Therefore

$$
\left.\frac{\partial W_{0}(x)}{\partial \mathbf{n}_{x}}\right|_{x \in \gamma^{+}}=0
$$

Joining (3.11), (3.12), (3.14), we obtain that the matching conditions hold:

$$
\left.W_{0}(x)\right|_{x \in \gamma^{+}}=\left.W_{0}(x)\right|_{x \in \gamma^{-}},\left.\quad \frac{\partial W_{0}(x)}{\partial \mathbf{n}_{x}}\right|_{x \in \gamma^{+}}=\left.\frac{\partial W_{0}(x)}{\partial \mathbf{n}_{x}}\right|_{x \in \gamma^{-}} .
$$

Recall that $W_{0}(x)$ is twice continuously differentiable and obeys the Helmholtz equation in $\left(\bigcup_{n=1}^{N_{2}} \mathcal{D}_{n}^{2}\right) \backslash \gamma$. Thanks to the matching conditions $(3.15)$, the function $W_{0}(x)$ can be analytically continued across $\gamma$, because $\gamma$ is a set of removable singularities for $W_{0}(x)$. In other words, it can be shown with the help of (3.15) and the 3-rd Green's formula $[2,24]$ that $W_{0}(x) \in C^{2}\left(\mathcal{D}_{n}^{2}\right)\left(n=1, \ldots, N_{2}\right)$, and $W_{0}(x)$ satisfies the Helmholtz equation (2.1) everywhere in $\mathcal{D}_{n}^{2}$, in particular, on $\gamma_{n}$. As shown in (3.13), $W_{0}(x)$ is identically equal to zero in the subdomain of $\mathcal{D}_{n}^{2}$, because $\mathcal{D}_{n}^{*} \subset \mathcal{D}_{n}^{2}$. At the same time, $W_{0}(x)$ is analytic in $\mathcal{D}_{n}^{2}$ as a solution of the Helmholtz equation [23, Chapt. 4, Sect. 4.4, Corollary 4.4.1]. According to the method of analytic continuation we can prove that

$$
W_{0}(x) \equiv 0, \quad x \in \mathcal{D}_{n}^{2} \quad\left(n=1, \ldots, N_{2}\right) .
$$

REMARK. Indeed, we can show that $W_{0}\left(x^{0}\right)=0$ for any $x^{0} \in \mathcal{D}_{n}^{2}$. To prove this we connect $x^{0}$ and a fixed interior point of $\mathcal{D}_{n}^{*}$ by an arc lying in $\mathcal{D}_{n}^{2}$. Then we cover the arc by a finite number of balls (circles if $m=2$ ) that lie in $\mathcal{D}_{n}^{2}$. The center of the first ball (circle) is the mentioned interior point of $\mathcal{D}_{n}^{*}$. Then balls (circles) go to $x^{0}$, so that the center of each ball (circle) is contained in the previous one and belongs to the arc. The last ball (circle) contains $x^{0}$. Since $W_{0}(x)$ is analytic in $\mathcal{D}_{n}^{2}$ as a solution of the Helmholtz equation [23, Chapt. 4, Sect. 4.4, Corollary 4.4.1], we expand it in the convergence Taylor series in each ball (circle). The coefficients of the Taylor series coincide with derivatives of $W_{0}(x)$ in the origin. The Taylor expansion in the first ball (circle) is identically equal to zero, because $W_{0}(x) \equiv 0$ in the vicinity of its origin thanks to (3.13), and so all derivatives of $W_{0}(x)$ in the origin as well as Taylor coefficients are equal to zero. Then we sequentially show that expansions in all other balls (circles) are also equal to zero, since the vicinity of the origin of each ball (circle) lies in the previous ball (circle), where $W_{0}(x)$ is identically equal to zero. Consequently, $W_{0}\left(x^{0}\right)=0$ for any $x^{0} \in \mathcal{D}_{n}^{2}$.

Using (3.9), (3.16) and the smoothness of $W_{0}(x)$ ensured by the class $\mathbf{K}_{0}$ we obtain

$$
W_{0}(x) \equiv 0 \quad \text { in } \mathbb{R}^{m} \backslash \mathcal{D}^{1} \quad(m=2 \text { or } m=3) .
$$


Thus, the homogeneous problem $\mathbf{V}_{0}$ has only a trivial solution. Consequently, the inhomogeneous problem $\mathbf{V}_{0}$ has no more than one solution. The theorem is proved.

4. Integral equations and the solution of the problem. In the present section we obtain the solution of problem $\mathbf{V}_{0}$ in the form of potentials, the density of which obeys the uniquely solvable Fredholm equation of the second kind on the total boundary $\Gamma \cup \gamma$. As noted above, this solution of problem $\mathbf{V}_{0}$ is also a solution of problem $\mathbf{V}$.

To prove the existence theorem we impose the additional condition on the function $f(x)$ in $(2.2)$ :

$$
f(x) \in C^{1, \lambda}\left(\Gamma^{1}\right) \cap C^{0}\left(\Gamma^{2}\right), \quad \lambda \in(0,1] .
$$

We look for a solution of the problem $\mathbf{V}_{0}$ in the form:

$$
W[\mu](x)=\int_{\Gamma^{2} \cup \gamma} \mu(y) \Phi_{k}(x, y) d s_{y}+\int_{\Gamma^{1}} \mu(y)\left(\frac{\partial}{\partial \mathbf{n}_{y}}-i\right) \Phi_{k}(x, y) d s_{y},
$$

where $y=\left(y_{1}, \ldots, y_{n}\right) \in \Gamma \cup \gamma$ and $\Phi_{k}(x, y)$ is a fundamental solution of the Helmholtz equation $(2.1)$ in $\mathbb{R}^{m}$, so that

$$
\Phi_{k}(x, y)= \begin{cases}\frac{i}{4} \mathcal{H}_{0}^{(1)}(k|x-y|) & \text { if } m=2, k>0, \\ \frac{1}{4 \pi} \frac{\exp (i k|x-y|)}{|x-y|} & \text { if } m=3, k \geq 0 .\end{cases}
$$

Here and further on we assume that $k>0$ if $m=2$. By $\mathcal{H}_{0}^{(1)}(z)$ we denote the Hankel function of the 1-st kind and index zero $[16,24]$ :

$$
\mathcal{H}_{0}^{(1)}(z)=\frac{\sqrt{2} \exp (i z-i \pi / 4)}{\pi \sqrt{z}} \int_{0}^{\infty} \exp (-t) t^{-1 / 2}\left(1+\frac{i t}{2 z}\right)^{-1 / 2} d t .
$$

We look for the density $\mu(x)$ of the potential (4.2) in $C^{1, \omega}\left(\Gamma^{1}\right) \cap C^{0}\left(\Gamma^{2} \cup \gamma\right)$, where the Hölder exponent $\omega \in(0,1)$. According to the properties of potentials $[2,6,24,25]$ the function (4.2) belongs to the class $\mathbf{K}_{0}$ and satisfies all conditions of the problem $\mathbf{V}_{0}$ except for the boundary conditions on $\Gamma^{+}$and $\gamma^{-}$. To satisfy the boundary conditions we substitute (4.2) into (2.2) and (3.8), use the limit formulas for normal derivatives of a single layer potential $[2,6,24,25]$ and arrive at the following integral equations of the second kind for the density $\mu(x)$ :

$$
\begin{aligned}
& -\frac{1}{2} \mu(x)+\int_{\Gamma^{1}} \mu(y)\left(\frac{\partial}{\partial \mathbf{n}_{y}}-i\right) \Phi_{k}(x, y) d s_{y} \\
& \quad+\int_{\Gamma^{2} \cup \gamma} \mu(y) \Phi_{k}(x, y) d s_{y}=f(x), \quad x \in \Gamma^{1}, \\
& \frac{1}{2} \mu(x)+\int_{\Gamma^{2} \cup \gamma} \mu(y) \frac{\partial}{\partial \mathbf{n}_{x}} \Phi_{k}(x, y) d s_{y} \\
& +\int_{\Gamma^{1}} \mu(y) \frac{\partial}{\partial \mathbf{n}_{x}}\left(\frac{\partial}{\partial \mathbf{n}_{y}}-i\right) \Phi_{k}(x, y) d s_{y}=f(x), \quad x \in \Gamma^{2},
\end{aligned}
$$




$$
\begin{aligned}
-\frac{1}{2} \mu(x)+\int_{\Gamma^{2} \cup \gamma} & \mu(y)\left(\frac{\partial}{\partial \mathbf{n}_{x}}-i\right) \Phi_{k}(x, y) d s_{y} \\
& +\int_{\Gamma^{1}} \mu(y)\left(\frac{\partial}{\partial \mathbf{n}_{x}}-i\right)\left(\frac{\partial}{\partial \mathbf{n}_{y}}-i\right) \Phi_{k}(x, y) d s_{y}=0, \quad x \in \gamma .
\end{aligned}
$$

Set

$$
\delta\left(y, \Gamma^{1}\right)= \begin{cases}0 & \text { if } y \notin \Gamma^{1} \\ 1 & \text { if } y \in \Gamma^{1}\end{cases}
$$

Equations (4.3), (4.4) can be written in the form of one equation of the second kind on the whole boundary $\Gamma \cup \gamma$ :

$$
\frac{1}{2} \mu(x)+\int_{\Gamma \cup \gamma} \mu(y) \Omega_{k}(x, y) d s_{y}=f_{0}(x), \quad x \in \Gamma \cup \gamma,
$$

where

$$
\begin{aligned}
& \Omega_{k}(x, y)= \begin{cases}-\left(\delta\left(y, \Gamma^{1}\right)\left(\frac{\partial}{\partial \mathbf{n}_{y}}-i\right)+\left(1-\delta\left(y, \Gamma^{1}\right)\right)\right) \Phi_{k}(x, y) & \text { if } x \in \Gamma^{1}, \\
\frac{\partial}{\partial \mathbf{n}_{x}}\left[\left(\left(1-\delta\left(y, \Gamma^{1}\right)\right)+\delta\left(y, \Gamma^{1}\right)\left(\frac{\partial}{\partial \mathbf{n}_{y}}-i\right)\right) \Phi_{k}(x, y)\right] & \text { if } x \in \Gamma^{2}, \\
-\left(\frac{\partial}{\partial \mathbf{n}_{x}}-i\right)\left[\left(\left(1-\delta\left(y, \Gamma^{1}\right)\right)+\delta\left(y, \Gamma^{1}\right)\left(\frac{\partial}{\partial \mathbf{n}_{y}}-i\right)\right) \Phi_{k}(x, y)\right] & \text { if } x \in \gamma,\end{cases} \\
& f_{0}(x)= \begin{cases}f(x) & \text { if } x \in \Gamma, \\
0 & \text { if } x \in \gamma .\end{cases}
\end{aligned}
$$

Since $\Gamma^{1} \in C^{2, \lambda}, \Gamma^{2} \cup \gamma \in C^{2,0}$, the kernel in the integral equation (4.5) has a weak singularity, and the integral term in (4.5) is continuous on $\Gamma \cup \gamma$ in $x$ (see [2, 24]). Therefore, the integral operator in (4.5) maps $C^{0}(\Gamma \cup \gamma)$ into itself. Moreover, (4.5) is a Fredholm integral equation in $C^{0}(\Gamma \cup \gamma)$, because its kernel has a weak singularity [24].

Let us show that any solution of integral equation (4.5) in $C^{0}(\Gamma \cup \gamma)$ automatically belongs to $C^{1, \omega}\left(\Gamma^{1}\right) \cap C^{0}\left(\Gamma^{2} \cup \gamma\right)$ with $\omega \in(0,1]$. Indeed, let $\mu(x)$ be an arbitrary solution of the integral equation (4.5) in $C^{0}(\Gamma \cup \gamma)$, i.e., $\mu(x)$ obeys (4.3), (4.4). The second integral term in (4.3a) is infinitely differentiable in $x$, since it does not have a singularity if $x=y$. According to [2, Theorem 2.15], the first integral in (4.3a) is a Hölder function in $x$ on $\Gamma^{1}$. It follows from the identity (4.3a) for $\mu(x)$ that $\mu(x)$ is a Hölder function in $x$ on $\Gamma^{1}$ also (here we take into account condition (4.1b)). Using [2, Theorem $2.22]$ we verify that the first integral term in (4.3a) belongs to $C^{1, \omega_{0}}\left(\Gamma^{1}\right)$ in $x$ for some $\omega_{0} \in(0,1)$. Proceeding from the identity (4.3a) for $\mu(x)$ and taking into account (4.1) we obtain $\mu(x) \in C^{1, \omega}\left(\Gamma^{1}\right)$, where $\omega=\min \left\{\omega_{0}, \lambda\right\}, 0<\omega<1$.

So, any solution of equation (4.5) in $C^{0}(\Gamma \cup \gamma)$ automatically belongs to $C^{1, \omega}\left(\Gamma^{1}\right) \cap$ $C^{0}\left(\Gamma^{2} \cup \gamma\right)$ with $\omega \in(0,1)$. The potential $W[\mu](x)$ belongs to the class $\mathbf{K}_{0}$ and satisfies all conditions of the problem $\mathbf{V}_{0}$. We arrive at

LEmma. Let conditions (3.6), (4.1) hold. If $\mu(x) \in C^{0}(\Gamma \cup \gamma)$ obeys the Fredholm equation (4.5), then 
1) $\mu(x) \in C^{1, \omega}\left(\Gamma^{1}\right) \cap C^{0}\left(\Gamma^{2} \cup \gamma\right)$ for some $\omega \in(0,1)$;

2) the potential (4.2) is a solution of the problem $\mathbf{V}_{0}$.

REMARK. The lemma is true for any $k \geq 0$ in a $3-\mathrm{D}$ case and for any $k>0$ in a 2-D case, i.e., we do not require that $k \in \mathcal{I}$ in the lemma.

Thus, below we look for a solution of equation (4.5) in $C^{0}(\Gamma \cup \gamma)$.

Assuming that condition (3.6) holds, we will show that the homogeneous Fredholm equation (4.5) has only the trivial solution for any $k \in \mathcal{I}$. Let $\mu^{0}(x)$ be a solution of the homogeneous equation (4.5). Then it obeys the homogeneous equations (4.3), (4.4). We substitute $\mu^{0}(x)$ in (4.2) and consider a function $W\left[\mu^{0}\right](x)$. On the basis of the lemma, $W\left[\mu^{0}\right](x)$ is a solution of the homogeneous problem $\mathbf{V}_{0}$. According to Theorem 2 , since condition (3.6) holds, this problem has only a trivial solution for any $k \in \mathcal{I}$, and we obtain

$$
W\left[\mu^{0}\right](x) \equiv 0 \quad \text { in } \mathbb{R}^{m} \backslash \mathcal{D}^{1} \quad(m=2 \text { or } m=3) .
$$

Recall that $W\left[\mu^{0}\right](x)$ belongs to the class $\mathbf{K}_{0}$, and so $W\left[\mu^{0}\right](x) \in C^{0}\left(\overline{\mathbb{R}^{m} \backslash \mathcal{D}^{1}}\right) \cap C^{2}\left(\mathbb{R}^{m} \backslash\right.$ $\left.\left(\mathcal{D}^{1} \cup \Gamma \cup \gamma\right)\right)$. Using the jump formulas [2, 6, 24, 25] for the normal derivatives of the single layer potential on $\Gamma^{2}$ and $\gamma$, we obtain

$$
\begin{gathered}
\left.\frac{\partial}{\partial \mathbf{n}_{x}} W\left[\mu^{0}\right](x)\right|_{x \in\left(\Gamma^{2}\right)^{+}}-\left.\frac{\partial}{\partial \mathbf{n}_{x}} W\left[\mu^{0}\right](x)\right|_{x \in\left(\Gamma^{2}\right)^{-}}=\left.\mu^{0}(x)\right|_{x \in \Gamma^{2}}=0 \\
\left.\frac{\partial}{\partial \mathbf{n}_{x}} W\left[\mu^{0}\right](x)\right|_{x \in \gamma^{+}}-\left.\frac{\partial}{\partial \mathbf{n}_{x}} W\left[\mu^{0}\right](x)\right|_{x \in \gamma^{-}}=\left.\mu^{0}(x)\right|_{x \in \gamma}=0 .
\end{gathered}
$$

Consequently, $\mu^{0}(x) \equiv 0$ for $x \in \Gamma^{2} \cup \gamma$ and $\mu^{0}(x)$ satisfies the following homogeneous equation on $\Gamma^{1}$ :

$$
-\frac{1}{2} \mu^{0}(x)+\int_{\Gamma^{1}} \mu^{0}(y)\left(\frac{\partial}{\partial \mathbf{n}_{y}}-i\right) \Phi_{k}(x, y) d s_{y}=0, \quad x \in \Gamma^{1} .
$$

To study this equation using the Fredholm alternative we consider the adjoint integral equation

$$
-\frac{1}{2} \nu^{0}(x)+\int_{\Gamma^{1}} \nu^{0}(y)\left(\frac{\partial}{\partial \mathbf{n}_{x}}+i\right) \bar{\Phi}_{k}(x, y) d s_{y}=0, \quad x \in \Gamma^{1}
$$

where

$$
\bar{\Phi}_{k}(x, y)= \begin{cases}-\frac{i}{4} \mathcal{H}_{0}^{(2)}(k|x-y|) & \text { if } m=2, k>0 \\ \frac{1}{4 \pi} \frac{\exp (-i k|x-y|)}{|x-y|} & \text { if } m=3, k \geq 0\end{cases}
$$

is a fundamental solution for equation (2.1). Below we suppose that $\nu^{0}(x)$ is an arbitrary solution of the homogeneous equation $(4.8)$ in $C^{0}\left(\Gamma^{1}\right)$. Then the single layer potential

$$
v(x)=v\left[\nu^{0}\right](x)=\int_{\Gamma^{1}} \nu^{0}(y) \bar{\Phi}_{k}(x, y) d s_{y} \in C^{0}\left(\mathbb{R}^{m}\right) \cap C^{2}\left(\mathbb{R}^{m} \backslash \Gamma^{1}\right)
$$


obeys the Helmholtz equation (2.1) in $\mathbb{R}^{m} \backslash \Gamma^{1}$ and meets the radiation conditions at infinity:

$$
v=O\left(|x|^{(1-m) / 2}\right), \quad \frac{\partial v}{\partial|x|}+i k v=o\left(|x|^{(1-m) / 2}\right), \quad|x| \rightarrow \infty .
$$

In addition, $\partial v / \partial \mathbf{n}_{x}$ exists on $\left(\Gamma^{1}\right)^{+}$and $\left(\Gamma^{1}\right)^{-}$as a uniform (with respect to $x \in \Gamma^{1}$ ) limit in the normal direction. The potential $v(x)$ satisfies the interior impedance problem in $\mathcal{D}_{n}^{1}\left(n=1, \ldots, N_{1}\right)$ :

$$
\begin{aligned}
& \Delta v+k^{2} v=0 \quad \text { in } \mathcal{D}_{n}^{1} \\
& \frac{\partial v}{\partial \mathbf{n}_{x}}+i v=0
\end{aligned}
$$

where (4.10) holds since (4.8) is true. Consider the energy equality for equation (2.1) in $\mathcal{D}_{n}^{1}\left(n=1, \ldots, N_{1}\right)$ :

$$
-\|\nabla v\|_{L_{2}\left(\mathcal{D}_{n}^{1}\right)}^{2}+k^{2}\|v\|_{L_{2}\left(\mathcal{D}_{n}^{1}\right)}^{2}=\int_{\left(\Gamma_{n}^{1}\right)^{-}} \bar{v}(x) \frac{\partial v(x)}{\partial \mathbf{n}_{x}} d s=-i \int_{\left(\Gamma_{n}^{1}\right)^{-}}|v(x)|^{2} d s .
$$

This equality can be derived by the technique of equidistant surfaces (curves) [24]. Taking the imaginary part in (4.11) we obtain

$$
\int_{\left(\Gamma_{n}^{1}\right)^{-}}|v(x)|^{2} d s=0 \quad\left(n=1, \ldots, N_{1}\right) .
$$

Consequently,

$$
\left.v(x)\right|_{\left(\Gamma_{n}^{1}\right)^{-}}=\left.v(x)\right|_{\Gamma_{n}^{1}}=\left.v(x)\right|_{\left(\Gamma_{n}^{1}\right)^{+}}=0 \quad\left(n=1, \ldots, N_{1}\right),
$$

because the single layer potential is continuous across $\Gamma^{1}$. From (4.10) we have

$$
\left.\frac{\partial v}{\partial \mathbf{n}_{x}}\right|_{\left(\Gamma_{n}^{1}\right)^{-}}=0 \quad\left(n=1, \ldots, N_{1}\right) \text {. }
$$

Using (4.12) we verify that $v(x)$ obeys the following homogeneous Dirichlet problem for the equation (2.1) in the exterior domain $\mathbb{R}^{m} \backslash \mathcal{D}^{1}$ :

$$
\begin{gathered}
\Delta v+k^{2} v=0 \quad \text { in } \mathbb{R}^{m} \backslash \mathcal{D}^{1}, \\
\left.v\right|_{\left(\Gamma^{1}\right)^{+}}=0, \\
v=O\left(|x|^{(1-m) / 2}\right), \quad \frac{\partial v}{\partial|x|}+i k v=o\left(|x|^{(1-m) / 2}\right), \quad|x| \rightarrow \infty .
\end{gathered}
$$

One can prove with the help of energy equalities and the Rellich lemma [2] that this problem has only the trivial solution $v(x) \equiv 0$ in $\mathbb{R}^{m} \backslash \mathcal{D}^{1}$. Thanks to the existence of a uniform normal derivative of $v(x)$ on $\left(\Gamma^{1}\right)^{+}$we obtain

$$
\left.\frac{\partial v}{\partial \mathbf{n}_{x}}\right|_{\left(\Gamma^{1}\right)^{+}}=0
$$

Using (4.13), (4.14) and the jump relation for the single layer potential, we obtain

$$
\left.\frac{\partial v}{\partial \mathbf{n}_{x}}\right|_{\left(\Gamma^{1}\right)^{+}}-\left.\frac{\partial v}{\partial \mathbf{n}_{x}}\right|_{\left(\Gamma^{1}\right)^{-}}=\nu^{0}(x)=0, \quad x \in \Gamma^{1} .
$$


Hence, $\nu^{0}(x) \equiv 0$ on $\Gamma^{1}$ and the homogeneous equation (4.8) has only a trivial solution. Proceeding from the Fredholm alternative, the adjoint homogeneous equation (4.7) has only a trivial solution also, i.e., $\mu^{0}(x) \equiv 0$ on $\Gamma^{1}$. Therefore $\mu^{0}(x) \equiv 0$ on $\Gamma \cup \gamma$.

Thus, assuming that condition (3.6) holds, we have proved that the homogeneous Fredholm integral equation (4.5) has only a trivial solution for any $k \in \mathcal{I}$. According to the Fredholm alternative, the inhomogeneous equation (4.5) is uniquely solvable with these assumptions for any $f_{0}(x) \in C^{0}(\Gamma \cup \gamma)$. We arrive at

TheOREm 3. Let condition (3.6) hold. If $k \in \mathcal{I}$, then the Fredholm integral equation (4.5) has a unique solution $\mu(x) \in C^{0}(\Gamma \cup \gamma)$ for any $f_{0}(x) \in C^{0}(\Gamma \cup \gamma)$, in particular, for any $f(x) \in C^{0}(\Gamma)$ in (4.6). If, in addition, condition (4.1) holds, then the solution $\mu(x)$ belongs to $C^{1, \omega}\left(\Gamma^{1}\right) \cap C^{0}\left(\Gamma^{2} \cup \gamma\right)$ for some $\omega \in(0,1)$.

Recall that condition (3.6) follows from (3.7).

Corollary 1. Let condition (3.7) hold. If $k \in\left[0, k_{0}\right]$ in case $m=3$ and $k \in\left(0, k_{0}\right]$ in case $m=2$, then the Fredholm equation (4.5) has a unique solution $\mu(x) \in C^{0}(\Gamma \cup \gamma)$ for any $f_{0}(x) \in C^{0}(\Gamma \cup \gamma)$, in particular, for any $f(x) \in C^{0}(\Gamma)$ in (4.6). If, in addition, condition (4.1) holds, then the solution $\mu(x)$ belongs to $C^{1, \omega}\left(\Gamma^{1}\right) \cap C^{0}\left(\Gamma^{2} \cup \gamma\right)$ for some $\omega \in(0,1)$.

The last statement of Theorem 3 and Corollary 1 follows from the lemma. From Theorem 3 and the lemma we obtain the solvability theorem for problem $\mathbf{V}_{0}$.

TheOREM 4. If conditions (3.6), (4.1) hold, then for any $k \in \mathcal{I}$ the solution of problem $\mathbf{V}_{0}$ exists and is given by a potential (4.2), where $\mu(x)$ is a unique solution of the Fredholm integral equation (4.5), ensured by Theorem 3 .

As noted above, any solution of problem $\mathbf{V}_{0}$ satisfies problem $\mathbf{V}$. Therefore, the solution of problem $\mathbf{V}_{0}$ constructed in Theorem 4 satisfies problem $\mathbf{V}$.

TheOREm 5. If conditions (3.6), (4.1) hold, then for any $k \in \mathcal{I}$ the solution of problem $\mathbf{V}$ exists and is given by the potential $(4.2)$, where $\mu(x)$ is a unique solution of the Fredholm integral equation (4.5), ensured by Theorem 3 .

Corollary 2. Let assumptions (3.7), (4.1) hold. Then for any $k \in\left[0, k_{0}\right]$ in case $m=3$ and for any $k \in\left(0, k_{0}\right]$ in case $m=2$, the solution of problem $\mathbf{V}$ is given by (4.2), where $\mu(x)$ is a unique solution of the Fredholm integral equation (4.5) ensured by Corollary 1.

Note that condition (3.7) can always be satisfied.

Theorem 5 and Corollary 2 are the main results of the present paper. Basing on the method of Fredholm integral equations, we proved the solvability of problem $\mathbf{V}$ for $\Gamma^{1} \in C^{2, \lambda}, \Gamma^{2} \in C^{2,0}, f(x) \in C^{1, \lambda}\left(\Gamma^{1}\right) \cap C^{0}\left(\Gamma^{2}\right)$. In fact, our proof is valid for any positive $k$, since $k_{0}$ can be taken as large as necessary. The method of hypersingular integral equations on $\Gamma^{2}$ presented in $[2,12,17]$ does not apply, since the normal derivative of the double layer potential used in this method may not exist under our conditions. Recall that in the method of a hypersingular integral equation we look for a solution of problem $\mathbf{V}$ on $\Gamma^{2}$ as a sum of single and double layer potentials. 
The basic idea of our method is such that we introduce the interior boundary inside interior domains (scatterers) bounded by $\Gamma^{2}$ and reduce problem $\mathbf{V}$ to the uniquely solvable Fredholm equation on the whole boundary. The solution of the problem is represented in the form of potentials on the whole boundary. From the physical standpoint, the single layer potential defined on interior boundaries can be considered as distributed sources placed inside interior domains (scatterers) instead of the infinite number of point sources used in $[4,5,21,22]$.

The advantage of our approach is that the uniquely solvable Fredholm integral equation (4.5) can be computed by standard codes, i.e., by discretization and inversion of a matrix. Since our method holds for any $k \in\left[0, k_{0}\right]$ in a 3 -D case and for any $k \in\left(0, k_{0}\right]$ in a $2-\mathrm{D}$ case, where $k_{0}$ is an arbitrary positive number, problem $\mathbf{V}$ can be computed for different $k$ without any changes in a computational scheme.

Acknowledgment. The research was partially supported by the RFBR grant 99-0101063

\section{REFERENCES}

[1] V. Ya. Arsenin, Methods of Mathematical Physics, Nauka, Moscow, 1974

[2] D. Colton and R. Kress, Integral Equation Methods in Scattering Theory, John Wiley and Sons, New York, 1983

[3] J. Giroire and J. C. Nedelec, Numerical solution of an exterior Neumann problem using a double layer potential, Math. Comp. 32, 973-990 (1978)

[4] D. S. Jones, Integral equations for the exterior acoustic problem, Quart. J. Mech. Appl. Math. 27, 129-142 (1974)

[5] D. S. Jones, Methods in Electromagnetic Wave Propagation, Oxford University Press, Oxford, 1994

[6] P. A. Krutitskii, Dirichlet problem for the Helmholtz equation outside cuts in a plane, Comput. Math. Math. Phys. 34, 1073-1090 (1994)

[7] P. A. Krutitskii, Neumann problem for the Helmholtz equation outside cuts in a plane, Comput. Math. Math. Phys. 34, 1421-1431 (1994)

[8] P. A. Krutitskii, The mixed problem for the Helmholtz equation in a multiply connected region, Comput. Math. Math. Phys. 36, 1087-1095 (1996)

[9] P. A. Krutitskii, The Neumann problem on wave propagation in a 2-D external domain with cuts, J. Math. Kyoto Univ. 38, 439-452 (1998)

[10] P. A. Krutitskii, Wave propagation in a 2-D external domain bounded by closed and open curves, Nonlinear Analysis, Theory, Methods and Applications 32, 135-144 (1998)

[11] R. Kussmaul, Ein numerische Verfahren zur Lösung des Neumannschen Aussenraumproblems für die Helmholtzsche Schwingungsgleichung, Computing 4, 246-273 (1969)

[12] R. Leis, Vorlesungen über partielle Differentialgleichungen zweiter Ordnung, Bibliographisches Instit, Mannheim, 1967

[13] I. K. Lifanov, Singular Integral Equations and Discrete Vortices, VSP, Utrecht, 1996

[14] W. L. Meyer, W. A. Bell, B. T. Zinn, and M. P. Stallybrass, Boundary integral solutions of three dimensional acoustic radiation problems, J. Sound and Vibration 59, 245-262 (1978)

[15] J. C. Nedelec, Curved finite element methods for the solution of singular integral equations on surfaces in $\mathbb{R}^{3}$, Comput. Math. Appl. Mech. Engrg. 8, 61-80 (1976)

[16] A. F. Nikiforov and V. B. Uvarov, Special Functions of Mathematical Physics, Birkhäuser, Basel, 1988

[17] O. I. Panich, To a problem on solvability of exterior boundary value problems for wave equation and Maxwell equations, Uspekhi Mathemat. Nauk. 20, 221-226 (1965)

[18] S. Prössdorf and B. Silbermann, Numerical Analysis for Integral and Related Operator Equations, Academie-Verlag, Berlin, 1991 
[19] S. Prössdorf and J. Saranen, A fully discrete approximation method for the exterior Neumann problem of the Helmholtz equation, Zeitschrift für Analysis und ihre Anwendungen 13, 683-695 (1999)

[20] C. Ruland, Ein Verfahren zur Lösung von $\left(\Delta+k^{2}\right) U=0$ in Assengebieten mit Ecken, Applicable Analysis 7, 69-79 (1978)

[21] F. Ursell, On the exterior problems of acoustics, Proc. Cambridge Philos. Soc. 74, 117-125 (1973)

[22] F. Ursell, On the exterior problems of acoustics II, Proc. Cambridge Philos. Soc. 84, 545-548 (1978)

[23] L. Hörmander, Linear Partial Differential Operators, Springer-Verlag, Berlin, 1963

[24] V. S. Vladimirov, Equations of Mathematical Physics, Marcel Dekker, New York, 1971

[25] C. H. Wilcox, Scattering theory for the d'Alembert equation in the exterior domains, Lecture Notes in Math. 422, Springer, Berlin, 1975 